\title{
Application of the Cloude-Pottier decomposition to weather radar signatures
}

\author{
M. Galletti ${ }^{1}$, M. Chandra ${ }^{1}$, E. Pottier ${ }^{2}$, and A. Ghorbani ${ }^{1, *}$ \\ ${ }^{1}$ TU-Chemnitz, Department of Microwave Engineering and Photonics, 09126 Chemnitz, Germany \\ ${ }^{2}$ Université de Rennes 1, IETR, Department Image and Remote Sensing, F-35042 Rennes, France \\ *TU-Chemnitz, Department of Microwave Engineering and Photonics, on sabbatical leave from Amir Kabir \\ University, Teheran, Iran
}

\begin{abstract}
In this paper we apply the Cloude-Pottier decomposition to Weather Radar Signatures. First, we present the results of a simulation carried out at the Chemnitz University of Technology and give the expected $\mathrm{H}-\alpha$ values for different rain intensities. A comparison with standard radarmeteorological variables is also given. Then, first ever images of Entropy and Anisotropy are presented for clouds and precipitation. Experimental Data are from the POLDIRAD Weather Facility in Oberpfaffenhofen, Germany.
\end{abstract}

\section{Introduction}

Since the pioneering works of Chandrasekhar and Huynen, Target Decomposition Theorems have been devoloped enormously and have given rise to a wide range of applications. A field that has not yet taken advantage of these techniques is radar meteorology, probably because of the shortage of fully polarimetric datasets. The experimental data employed in this study come from the DLR Polarisation Diversity Weather Radar whose TYPE 18 was recently made available with new IDL code written at the Chemnitz University of Technology.

By measuring the time series of $\mathrm{S}$ matrices and after correcting them for the Doppler phase shift, it is possible to proceed to second order statistics analysis.

Two second order descriptors are the well known covariance and coherency matrices, obtainable by means of different vectorial operations. Traditionally, radar meteorologists use the lexicographic basis.

$\left[\psi_{L}\right]=\left\{2\left[\begin{array}{ll}1 & 0 \\ 0 & 0\end{array}\right], 2\left[\begin{array}{ll}0 & 1 \\ 0 & 0\end{array}\right] 2\left[\begin{array}{ll}0 & 0 \\ 1 & 0\end{array}\right] 2\left[\begin{array}{ll}0 & 0 \\ 0 & 1\end{array}\right]\right\}$

Correspondence to: $\mathrm{M}$. Galletti

(michele@infotech.tu-chemnitz.de)
In the monostatic case, the $\mathrm{S}$ matrix is supposed to be symmetric and the second and third matrices of the above list melt into the following

$\psi_{L 1}\left[\begin{array}{ll}0 & 1 \\ 1 & 0\end{array}\right]$

Given the lexicographic basis, it is possible to build the lexicographic target vector.

$$
\begin{aligned}
& {[S]=\left[\begin{array}{ll}
S_{H H} & S_{H V} \\
S_{V H} & S_{V V}
\end{array}\right] \Rightarrow} \\
& \Rightarrow \underline{\Omega}=V([S])=\frac{1}{2} \text { Trace }\left([S]\left[\psi_{L}\right]\right)
\end{aligned}
$$

For the monostatic case, this yields

$\underline{\Omega}=\left[\begin{array}{c}S_{H H} \\ \sqrt{2} S_{H V} \\ S_{V V}\end{array}\right]$.

We can now write the covariance matrix and, after averaging over a certain number of samples that are supposed to be representative of the same weather target, we can derive all radar meteorological variables.

$[C]=\underline{\Omega} \cdot \underline{\Omega}^{t *}=$

$\left[\begin{array}{ccc}\left|S_{H H}\right|^{2} & \sqrt{2} S_{H H} S_{H V}^{*} & S_{H H} S_{V V}^{*} \\ \sqrt{2} S_{H V} S_{H H}^{*} & 2\left|S_{H V}\right|^{2} & \sqrt{2} S_{H V} S_{V V}^{*} \\ S_{V V} S_{H H}^{*} & \sqrt{2} S_{V V} S_{H V}^{*} & \left|S_{V V}\right|^{2}\end{array}\right]$

From the main diagonal we obtain $\mathrm{Z}_{H H}, \mathrm{Z}_{V V}, \mathrm{Z}_{D R}$, $\mathrm{LDR}_{1}$, and $\mathrm{LDR}_{2}$. From the covariance between $\mathrm{S}_{H H}$ and $\mathrm{S}_{V V}$ we get $\Phi_{D P}, \mathrm{~K}_{D P}$ and $\rho_{H V}$.

Another approach, typically used in Polarimetric Sar, is to take the set of Pauli matrices.

$\left[\psi_{P}\right]=\left\{\sqrt{2}\left[\begin{array}{ll}1 & 0 \\ 0 & 1\end{array}\right], \sqrt{2}\left[\begin{array}{cc}1 & 0 \\ 0 & -1\end{array}\right] \sqrt{2}\left[\begin{array}{ll}0 & 1 \\ 1 & 0\end{array}\right] \sqrt{2}\left[\begin{array}{cc}0 & -i \\ i & 0\end{array}\right]\right\}$

Instead of the lexicographic scattering vector, the Pauli scattering vector can be built:

$$
\begin{aligned}
& {[S]=\left[\begin{array}{ll}
S_{H H} & S_{H V} \\
S_{V H} & S_{V V}
\end{array}\right] \Rightarrow} \\
& \Rightarrow \underline{k}=V([S])=\frac{1}{2} \text { Trace }\left([S]\left[\psi_{P}\right]\right)
\end{aligned}
$$


For the monostatic case, we simply drop the fourth of Pauli matrices, so that we write the Pauli scattering vector as

$\underline{k}=\frac{1}{\sqrt{2}}\left[\begin{array}{c}S_{H H}+S_{V V} \\ S_{H H}-S_{V V} \\ 2 S_{H V}\end{array}\right]$

In Eq. (9) we write the single look Coherency matrix T. After spatial averaging over different pixels supposed to be representative of the same distributed target, the averaged coherency matrix is the starting point for applying stochastic decomposition theorems.

$T=\underline{k} \cdot \underline{k}^{t *}$

\section{Theoretical background}

\section{- A. Cloude's Point Target Reduction Theorem}

As stated above, an S matrix (a coherent scattering mechanism) can be described by the complex vector $\underline{\mathrm{k}}$.

The Pauli scattering vector can be parametrized as

$\underline{k}=\left[\begin{array}{l}\mathrm{a} \\ \mathrm{b} \\ \mathrm{c}\end{array}\right]=|\underline{k}|\left[\begin{array}{c}\cos \alpha e^{i \varphi_{1}} \\ \sin \alpha \cos \beta e^{i \varphi_{2}} \\ \sin \alpha \sin \beta e^{i \varphi_{3}}\end{array}\right]=$

$|\underline{k}| e^{i \varphi}\left[\begin{array}{c}\cos \alpha \\ \sin \alpha \cos \beta e^{i \delta} \\ \sin \alpha \sin \beta e^{i \gamma}\end{array}\right]$

where $\varphi=\varphi_{1}, \delta=\varphi_{2}-\varphi_{1}, \gamma=\varphi_{3}-\varphi_{1}$.

The angles are defined as follows:

$\alpha$ target scattering mechanism $0 \leq \alpha \leq 90^{\circ}$

$\beta$ target orientation $0^{\circ} \leq \beta \leq 90^{\circ}$

$\phi, \delta, \gamma$ phase angles $-180^{\circ} \leq \varphi, \delta, \gamma \leq+180^{\circ}$.

Any coherent scattering mechanism can be transformed into another by plane rotations as shown in Eqs. (11) and (12).

$\underline{k}^{\prime}=\left[\begin{array}{ccc}1 & 0 & 0 \\ 0 & \cos \Delta \beta & -\sin \Delta \beta \\ 0 & \sin \Delta \beta & \cos \Delta \beta\end{array}\right] \underline{k}$

$\underline{k}^{\prime}=\left[\begin{array}{ccc}\cos \Delta \alpha & -\sin \Delta \alpha & 0 \\ \sin \Delta \alpha & \cos \Delta \alpha & 0 \\ 0 & 0 & 1\end{array}\right] \underline{k}$

This observation leads to Cloude's Point Target Reduction Theorem:

"Any Pauli scattering vector can be reduced to the identity by a series of three transformations as shown in Eq. (13)"

$$
\begin{aligned}
& \underline{e}=\left[\begin{array}{l}
1 \\
0 \\
0
\end{array}\right]=\left[\begin{array}{ccc}
\cos \alpha & \sin \alpha & 0 \\
-\sin \alpha & \cos \alpha & 0 \\
0 & 0 & 1
\end{array}\right] \\
& {\left[\begin{array}{ccc}
1 & 0 & 0 \\
0 & \cos \beta & \sin \beta \\
0 & -\sin \beta & \cos \beta
\end{array}\right] \times\left[\begin{array}{ccc}
e^{-i \varphi_{1}} & 0 & 0 \\
0 & e^{-i \varphi_{2}} & 0 \\
0 & 0 & e^{-i \varphi_{3}}
\end{array}\right] \underline{k} .}
\end{aligned}
$$

If the coherent target has some symmetry axis there is a reference frame where the $\mathrm{S}$ matrix is diagonal and an orientation can be defined. It can be shown that the $\beta$ angle is related to the physical orientation of the target around the radar line of sight. Indeed, if symmetric targets are illuminated, $\beta / 2$ is in some way representative of the absolute value of the canting angle.

The $\alpha$ angle has a range of $90^{\circ}$ and parametrizes a smooth variation between different scattering mechanisms. If it equals $0^{\circ}$ we have the first Pauli matrix whose physical counterpart is the isotropic scatterer. This can be regarded as a sphere, an isotropic surface or an odd-bounce scattering mechanism (a trihedral for example).

If $\alpha$ equals $90^{\circ}$ we have the second and third Pauli matrices, whose physical counterparts are the horizontal and $45^{\circ}$ tilted dihedral (or, more generally, even bounce scatterers at $0^{\circ}$ and $45^{\circ}$ ). From $0^{\circ}$ to $45^{\circ} \alpha$ parametrizes a transition to a fully isotropic scatterer (a sphere for example) to a fully anisotropic scatterer (a dipole). This range of scatterers can be called anisotropic surfaces. From $45^{\circ}$ to $90^{\circ}$ we have the transition from the dipole to the dihedrals. These scatterers can be called anisotropic dihedrals because the phase difference between the two diagonal terms of the $\mathrm{S}$ matrix is $180^{\circ}$ and their absolute values differ but for the dihedrals. Note that in the above parametrization $\alpha$ is decoupled from $\beta$, this means that $\alpha$ is able to detect a scattering mechanism regardless its orientation around the radar line of sight.

\section{- B. Generalized Stochastic Decomposition}

Weather radar as well as POLSAR targets are in general distributed targets. This implies that the space-time variability of the target makes every S-matrix a point in a multivariate statistical distribution (Gaussian, 0 mean). We resort then to average out the absolute phase of singular $\mathrm{S}$ matrices into a suitable second order mathematical descriptor that can be the covariance or the coherency matrix.

The coherency matrix $\mathrm{T}$ is always a hermitian matrix that can undergo an eigenvalue analysis.

$$
[T]=\left[U_{3}\right]\left[\begin{array}{ccc}
\lambda_{1} & 0 & 0 \\
0 & \lambda_{2} & 0 \\
0 & 0 & \lambda_{3}
\end{array}\right]\left[U_{3}\right]^{* T}
$$

with

$\left[U_{3}\right]=$

$\left[\begin{array}{ccc}\cos \alpha_{1} & \cos \alpha_{2} & \cos \alpha_{3} \\ \sin \alpha_{1} \cos \beta_{1} e^{i \delta_{1}} & \sin \alpha_{2} \cos \beta_{2} e^{i \delta_{2}} & \sin \alpha_{3} \cos \beta_{3} e^{i \delta_{3}} \\ \sin \alpha_{1} \sin \beta_{1} e^{i \gamma_{1}} & \sin \alpha_{2} \sin \beta_{2} e^{i \gamma_{2}} & \sin \alpha_{3} \sin \beta_{3} e^{i \gamma_{3}}\end{array}\right]$

and $\lambda_{1} \geq \lambda_{2} \geq \lambda_{3} \geq 0$.

This operation is such that an incoherent scattering process can be decomposed into 3 coherent scattering mechanisms.

Indeed, the spectral decomposition of the coherency matrix reads:

$\langle[T]\rangle=\lambda_{1}{\underline{u_{1}}}^{u_{1}}{ }^{*}+\lambda_{2} \underline{u}_{2} u_{2}^{*}+\lambda_{3} \underline{u}_{3} u_{3}^{*}$ 
Two interpretations have been given to this decomposition. The first one is the identification of the dominant scattering mechanism via the extraction of the largest eigenvalue.

The second interpretation with little more algebra distinguishes a pure target from a mixed target and unpolarised noise.

For our purposes however, the Cloude-Pottier decomposition will be more suitable.

\section{- C. Cloude-Pottier Decomposition}

From the parametrization of the $\mathrm{SU}(3)$ unitary matrix $\mathrm{U}_{3}$ we obtain a set of three orthogonal eigenvectors. Each of these principal components is representative of a coherent scattering mechanism.

The next step is the identification of a "mean scattering mechanism". This can be achieved through an eigenvalueweighted averaging as shown below.

First we define the weights

$P_{i}=\frac{\lambda_{i}}{\sum_{k=1}^{3} \lambda_{k}}$

And then the averaged parameters:

$\alpha=P_{1} \alpha_{1}+P_{2} \alpha_{2}+P_{3} \alpha_{3}$

$\bar{\beta}=P_{1} \beta_{1}+P_{2} \beta_{2}+P_{3} \beta_{3}$

$\bar{\gamma}=P_{1} \gamma_{1}+P_{2} \gamma_{2}+P_{3} \gamma_{3}$

$\bar{\delta}=P_{1} \delta_{1}+P_{2} \delta_{2}+P_{3} \delta_{3}$

With which we can define a unitary target vector of the mean scattering mechanism:

$\underline{u_{0}}=\left[\begin{array}{c}\cos (\underline{\alpha}) \\ \sin (\underline{\alpha}) \cos (\underline{\beta}) e^{i \underline{\delta}} \\ \sin (\underline{\alpha}) \sin (\underline{\beta}) e^{i \underline{\gamma}}\end{array}\right]$

considering the target magnitude,

$\underline{\lambda}=P_{1} \lambda_{1}+P_{2} \lambda_{2}+P_{3} \lambda_{3}$

We end up with the target vector of the mean coherent scattering mechanism representative of a stochastic scattering process.

$\underline{u_{0}}=\sqrt{\underline{\lambda}}\left[\begin{array}{c}\cos (\underline{\alpha}) \\ \sin (\underline{\alpha}) \cos (\underline{\beta}) e^{i \underline{\delta}} \\ \sin (\underline{\alpha}) \sin (\underline{\beta}) e^{i \underline{\gamma}}\end{array}\right]$

Besides eigenvector derived variables it is desirable to have some eigenvalue-derived descriptors.

$H=-\sum_{i=1}^{3} P_{i} \log _{3}\left(P_{i}\right)$
$A=\frac{\lambda_{2}-\lambda_{3}}{\lambda_{2}+\lambda_{3}}$
$\operatorname{Span}(\mathrm{T})=\sum \lambda_{\mathrm{i}}$

In Eq. (22) the first variable, named entropy, is a measure of how much the three equivalent coherent scattering mechanisms are mixed up in the stochastic scattering process. It can be regarded as a measure of the depolarizing properties of the observed target.

Anisotropy is a measure of the intensity difference between the two minor principal components and can be regarded as a measure of target homogeneity around the radar line of sight or, in other words, how asymmetric are the depolarizing properties of the observed medium.

The Span of the coherency matrix is a measure of the total backscattered power, namely co and cross polar powers at horizontal polarization plus the co and cross polar powers at vertical polarization.

\section{The raincloud simulation}

We now turn to the description and results of a simulation carried out at the Chemnitz University of Technology.

\section{- A. Description}

The simulated scenario consists of precipitation drops. No canting of the drops is assumed and only single scattering is modeled. No Mie effects are included and all formulas refer to Rayleigh scattering. No propagation effects are considered.

As input, the rain-rate (in $\mathrm{mm} / \mathrm{h}$ ) and the Drop Size Distribution (sometimes referred to as DSD) are requested. Two exponential DSDs can be chosen: Marshall and Palmer and Joss-Thunderstorm.

Given an exponential distribution,

$$
N(D)=N_{0} e^{-\Lambda D}
$$

a Marshall and Palmer has the following parameters:

$N_{0}=8000$

$\Lambda=4.1 \cdot R R^{-0.21}$

and a Joss-thunderstorm, more suitable for convective events, is given by:

$$
\begin{aligned}
& N_{0}=8000 \cdot R R^{0.37} \\
& \Lambda=3.8 \cdot R R^{-0.14}
\end{aligned}
$$

Here RR indicates the rain rate.

Given the DSD, a Pruppacher and Beard drop-flattening model is assumed. This takes into account the fact that drops, while falling, tend to assume an oblate shape and thus breaking target azymuthal symmetry. The largest the drop equivalent diameter, the higher the axis ratio. Drops whose equivalent diameter is less than $1 \mathrm{~mm}$ do not show oblateness. The flattening relation employed is derived from wind tunnel experiments and can be found in Pruppacher and Klett (1997).

The simulation output is the Covariance and Coherency matrices. From the first we compute all standard radarmeteorological variables and from the coherency matrix we extract eigenvector and eigenvalue derived variables. 


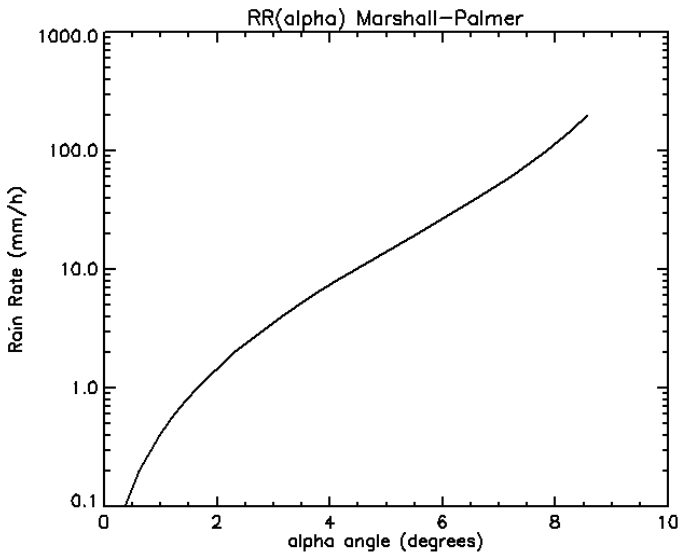

Fig. 1. Alpha in dependence of rain-rate (Marshall-Palmer).

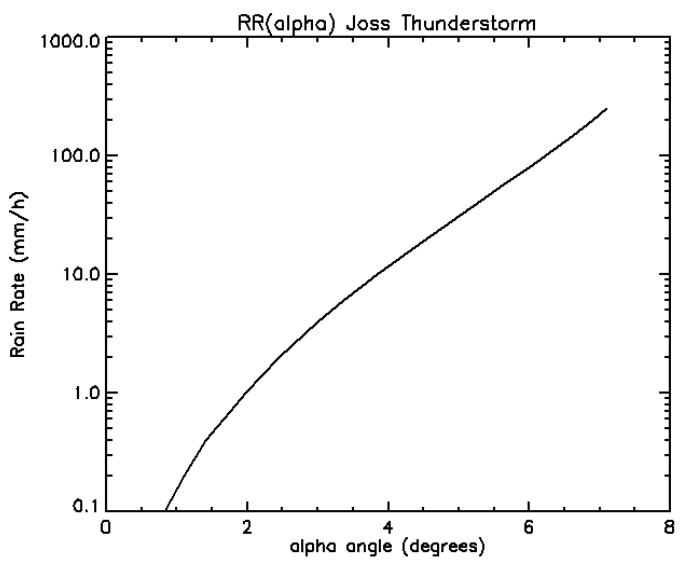

Fig. 2. Alpha in dependence of rain rate (Joss Thunderstorm).

Some formulas for the $\mathrm{C}$ and $\mathrm{T}$ matrix elements are given in Eq. (23) as an example.

$$
\begin{aligned}
C_{11} & =\int_{0}^{6} S_{h h}(D) \cdot S_{h h}(D)^{*} N(D) d D \\
T_{11} & =\int_{0}^{6}\left(S_{h h}(D)+S_{v v}(D)\right) \cdot\left(S_{h h}(D)+S_{v v}(D)\right)^{*} \cdot N(D) d D
\end{aligned}
$$

Here, $\mathrm{N}(\mathrm{D})$ is the drop size distribution and $\mathrm{S}_{H H}$ and $\mathrm{S}_{V V}$ are computed for an oblate, non canted spheroid of equivalent diameter $\mathrm{D}$.

\section{- B. Results}

If we think of a cloud of spheroids and only single scattering occurs, we can do the following observations.

Oblateness affects alpha but not beta. Mean canting angle affects beta but not alpha.

Variability in oblateness and canting angle affects entropy.

Some examples are given to get acquainted with the subject.

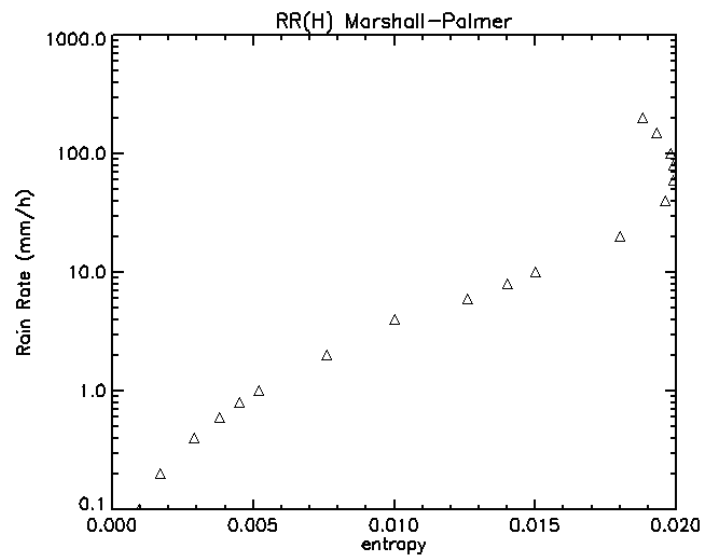

Fig. 3. Entropy in dependence of rain-rate (Marshall-Palmer).

If we think of a cloud of spheres of different size $\mathrm{H}$ will be zero because only one scattering mechanism occurs (the $S$ matrix is the same for all scatterers), $\alpha$ will be zero because of the scatterers isotropicity $\left(\mathrm{S}_{h h}=\mathrm{S}_{v v}\right)$ and in this case $\beta$ is basically undefined.

Let us now think of a cloud of same size, non canted, oblate spheroids with the same axis ratio. $\mathrm{H}$ will be zero because the scattering mechanism is the same for all scatterers, $\alpha$ will assume some value between $0^{\circ}$ and $45^{\circ}$, in the limit, $0^{\circ}$ if the spheroids are spheres and $45^{\circ}$ if they are dipoles.

Let us think of a cloud of oblate spheroids with same size and axis ratio as above, but this time with a distribution of canting angles. Now $\alpha$ will have the same value as above, $\beta$ will be the mean canting angle and $\mathrm{H}$ will increase accordingly to the features of the canting distribution (note that if all spheroids had the same canting angle $\mathrm{H}$ would be zero, even though the mean canting angle is not zero).

Our simulation models the realistic case where there is a distribution in the spheroids size (given by the DSD) and oblateness is dependent on size (Pruppacher and Beard flattening model).

Alpha should be between $0^{\circ}$ and $45^{\circ}$, and will probably have low values because the scattering mechanisms are still very sphere-like (low anisotropicity). $\mathrm{H}$ is sensitive to the spread in scattering mechanisms. In our model the spread is given by spread in oblateness, weighted with backscatter intensity because of the coupling with size.

Figure 1 and 2 show alpha as a function of rain-rate for the Marshall and Palmer and Joss-Thunderstorm distributions. As expected, being raindrops not far from sphere-like shapes alpha has small values. Furthermore, whatever the rain rate, alpha never takes on values greater than $9^{\circ}$. This small variability range implies that this variable cannot be helpful in discriminating rain intensities.

In Figs. 3 and 4 we have entropy values as a function of rain rates. The dispersion induced by the increase in rain intensity (oblateness dispersion) does not affect entropy sensitively. In this case too, its values are confined into a very 


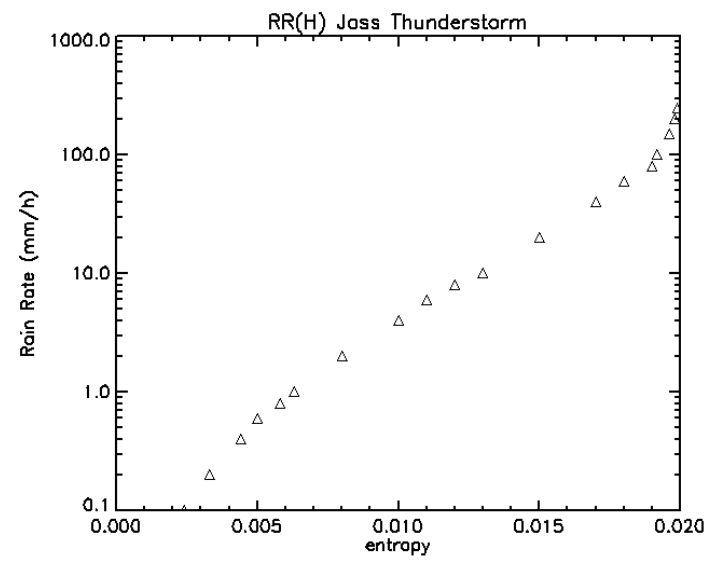

Fig. 4. Entropy in dependence of rain rate (Joss-Thunderstorm).

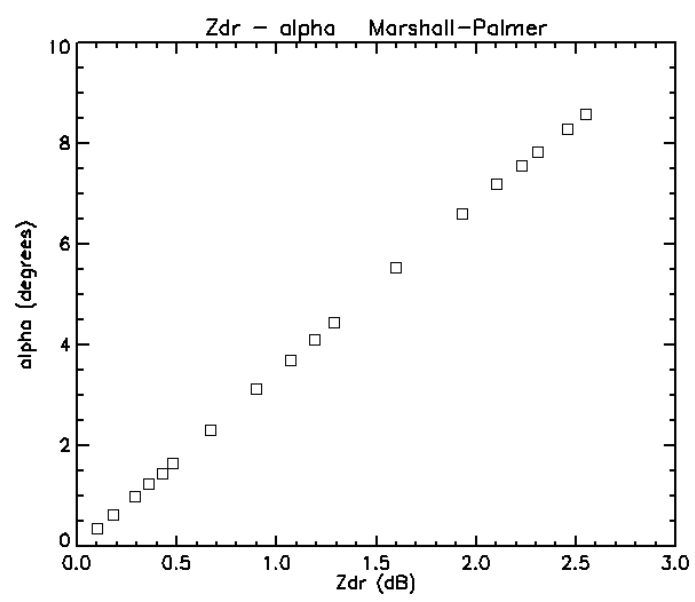

Fig. 5. DR vs. $\alpha$ (Marshall-Palmer) for increasing rain-rates (0.1$200 \mathrm{~mm} / \mathrm{h})$.

small range and any application for rain rate estimation appears not feasible.

In the Marshall and Palmer graph we can see that in the upper right corner entropy starts to decrease. Considering the exponential distributions and the flattening model employed it is reasonable to think that scattering mechanism dispersion saturates for high rain rates.

Indeed, the same thing would happen in the JossThunderstorm case if we plotted unreasonably high rain rates.

It is important to note that, whatever the DSD, these variables cannot discriminate between rain intensities.

In Figs. 5, 6, and 7 we give, for increasing rain rates (from 0.1 to $200 \mathrm{~mm} / \mathrm{h}$ ), a comparison between different variables. Figure 5 shows the dependence between $\alpha$ and $\mathrm{Z}_{D R}$. When observing rain, they are both sensitive to drop oblateness. One difference is that $\mathrm{Z}_{D R}$, being worked out in the lexicographic basis, is biased by drop canting, while $\alpha$ is independent from the scatterers' orientation. Figure 6 shows the $\mathrm{H}-\alpha$ plane. It is indeed a blow-up of a very small region in the

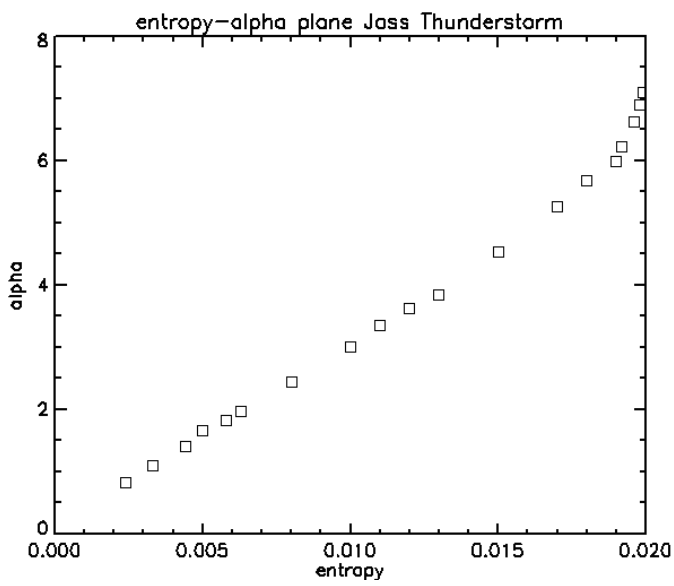

Fig. 6. $\mathrm{H}-\alpha$ plane for increasing rain rates $(0.1-200 \mathrm{~mm} / \mathrm{h})$.

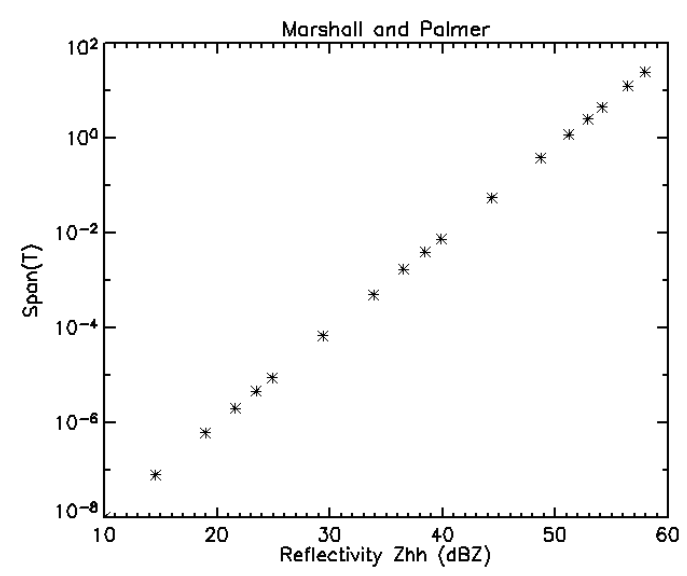

Fig. 7. Span of the coherency matrix vs. Reflectivity for increasing rain-rates $(0.1-200 \mathrm{~mm} / \mathrm{h})$.

lower-left corner of the plane where all points for different (simulated) rain rates tend to cluster.

In Fig. 7 is plotted a comparison between the Span of the coherency matrix and $\mathrm{Z}_{H H}$. In general, we can say that if $\mathrm{Z}_{H H}$ is representative of the horizontal backscattered power at $\mathrm{H}$-polarization, the span is sensitive to backscatter reflectivity at all polarisations.

\section{Experimental data}

We now consider some experimental data and see how the Cloude-Pottier Decomposition can play an important role in weather radar polarimetry.

\section{- A. Stratiform}

Stratiform events usually present simple standard patterns and we might like to identify some.

In Figs. 8 and 9, $\mathrm{Z}$ and $\mathrm{Z}_{D R}$ are given.

It is possible to identify the melting band at about $1 \mathrm{~km}$ height, with higher $Z_{D R}$ values and slightly higher $Z$ values. 


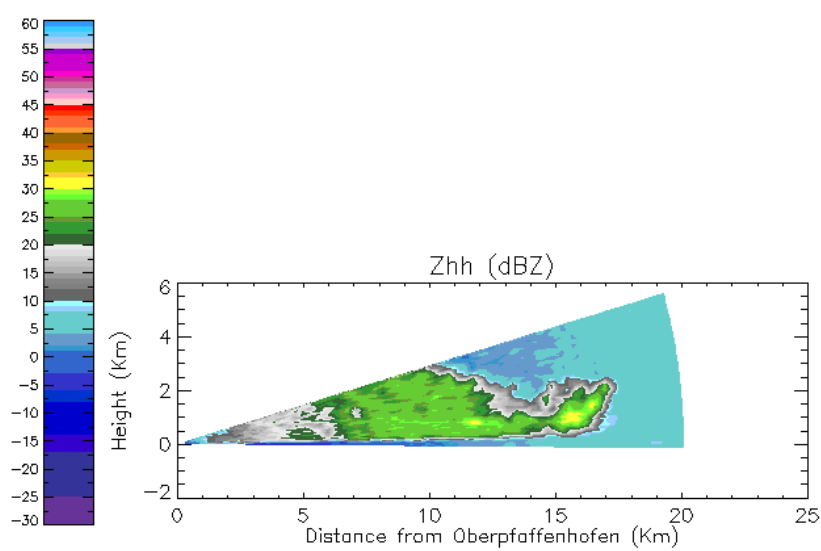

Fig. 8. Horizontal Reflectivity (stratiform).
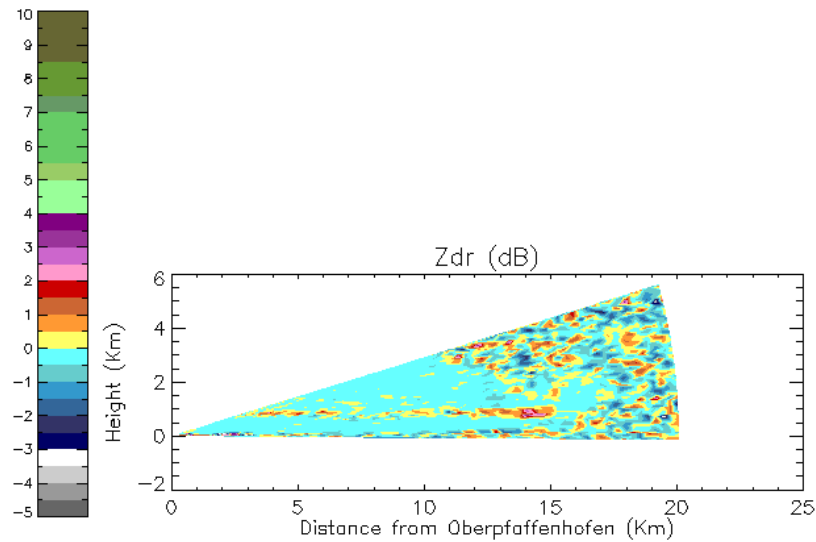

Fig. 9. Differential Reflectivity (stratiform).

We might reasonably expect to find snow above and rain below. $\mathrm{Z}_{D R}$ does not show positive values below the melting band and the relatively weak $Z$ values suggest some very light rain can be present. Outside the precipitation area atmospheric and hardware noise show up: $\mathrm{Z}$ falls to low values (below $10 \mathrm{~dB}$ ) and $\mathrm{Z}_{D R}$ flickers around $0 \mathrm{~dB}$.

Figure 10 shows the Entropy map of stratiform precipitation. The Entropy clearly highlights the melting band, with values ranging between 0.2 and 0.6 .

Below and above the bright band, clouds of drops or ice particles (light rain and snowflakes) take on low $\mathrm{H}$ values, between 0.0 and 0.2 .

As far as rain is concerned, this is in qualitative agreement with the simulation, and the assumption that only single scattering occurs turned to be reasonable (at least in this case where the observed event is not too severe).

Where noise dominates (noise can be regarded as random incoherent scattering), we expect all three equivalent coherent scattering mechanisms to have about the same intensities.

Entropy should then jump to 1 and Anisotropy (Fig. 11) should drop to 0 . This is what actually happens.

In low $\mathrm{H}$ areas, A must not be regarded as a meaningful parameter, being the ratio of noise over noise.

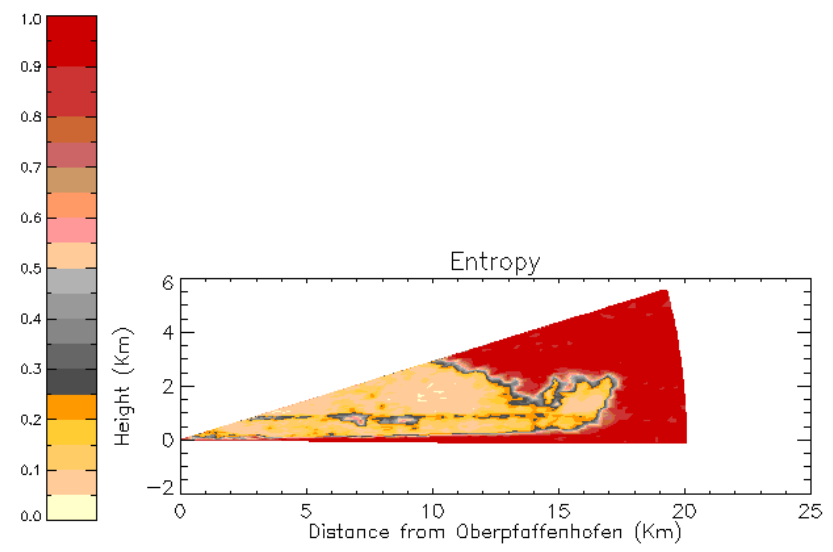

Fig. 10. Entropy (stratiform) $-\mathrm{H}$.

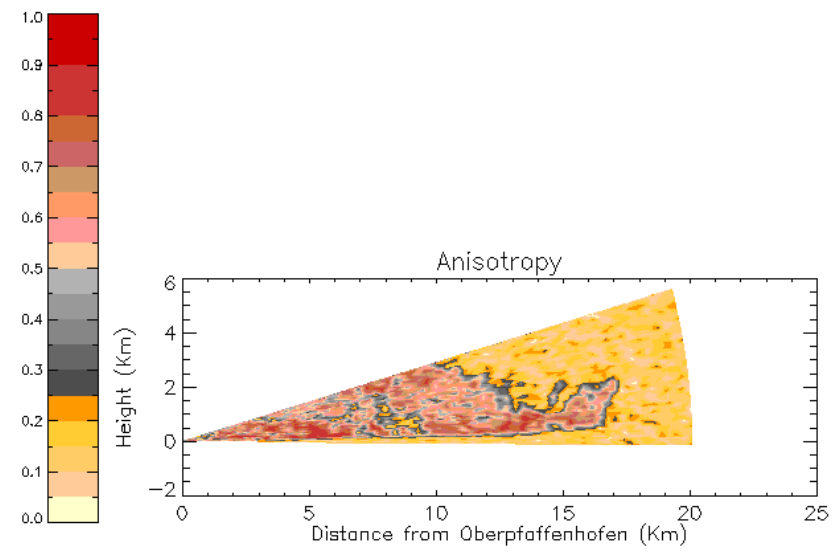

Fig. 11. Anisotropy (stratiform) - A.

In this case Anisotropy does not add significant information.

\section{- B. Convective}

We now turn to the analysis of a more complex event, characterised by a deep convective core with very high $\mathrm{Z}$ and low $\mathrm{Z}_{D R}$ values, probably indicating the presence of frozen hydrometeors like hail or graupel.

Figure 12 shows the Span of the coherency matrix. Being the range spread over some orders of magnitude, it is expressed in decibels. For this event, the Span map perfectly mirrors the reflectivity map, here not shown.

Figure 13 shows the Entropy map for the same event. Contrary to the stratiform event previously analysed, here weather signatures span over the whole 0-1 interval. Hailstones have a greater variability in shape than raindrops. This variability causes the backscattered wave (the sum of all fully polarised waves coming from each hydrometeor) to be less coherent than a wave given by the sum of waves backscattered by almost spherical raindrops. 

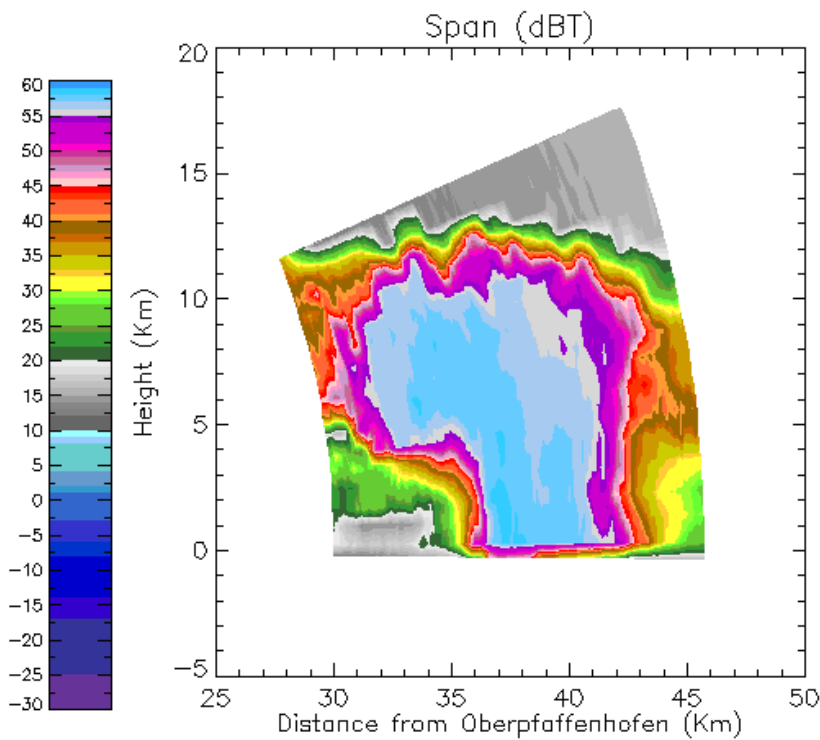

Fig. 12. Span (convective).

This means that, when dealing with convective cores, the depolarizing effects of big, irregularly shaped, frozen hydrometeors become visible.

Furthermore, when dealing with hailstones it is possible that volume scattering can contribute to the observed high $\mathrm{H}$ values.

Multiple scattering (cloud-ground-(cloud)) paths can also come into play when dealing with this type of events and different scattering mechanisms could show up.

How much all these scattering mechanisms contribute to the backscattered signal is an issue that needs to be addressed.

Only qualitative conclusions can be drawn at this stage. Yellow spots (low entropy) are present only in the outer parts of the convective core, where raindrops are most likely to be found and single scattering mechanisms are likely to prevail.

As we get closer to the storm's centre, $\mathrm{H}$ increases to high values probably because of hydrometeors' shape variability and perhaps because of volume scattering.

Figure 14 shows the Anisotropy map for the same convective event.

Disregarding low entropy areas, we might like to pay attention to the very centre of the convective core where this variable could give further insight on the severe phenomena harboured therein.

\section{Conclusions}

The eigenvalue-derived variables provided by the CloudePottier decomposition seem promising for the investigation of storms and precipitation. In particular, they might be helpful in the analysis of deep convective cores where depolarisation effects and multiple scattering may play a dominant role.

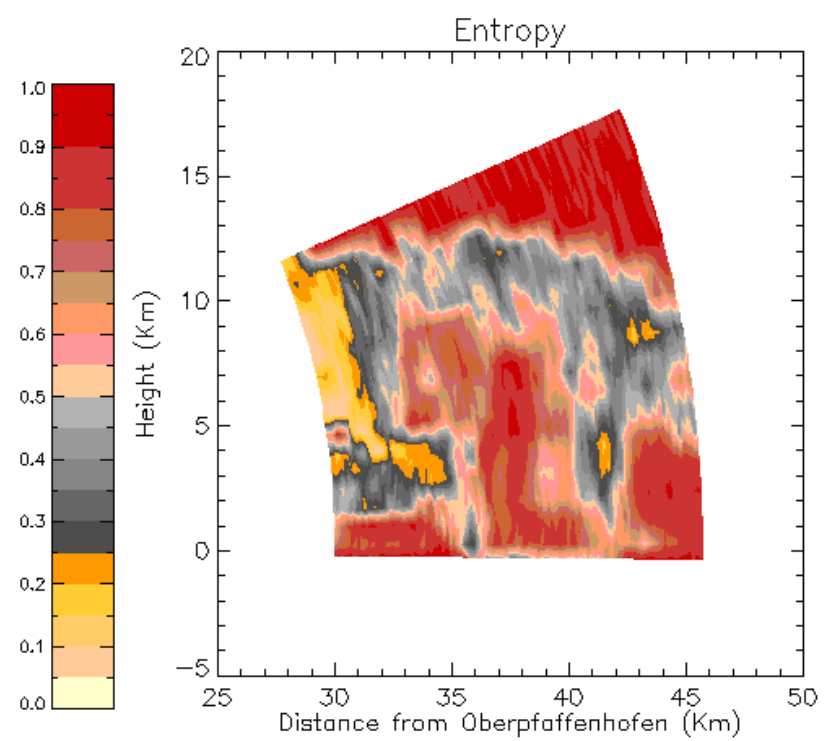

Fig. 13. Entropy (convective).

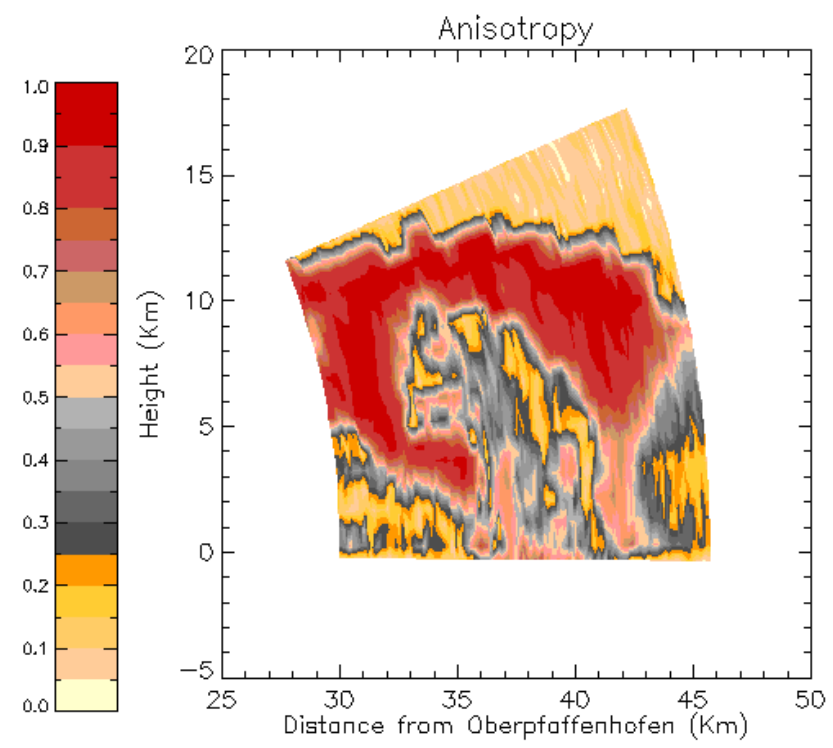

Fig. 14. Anisotropy (convective).

Target decomposition involves cross-polar terms. To get good results, we must ensure that cross-polar channels are not contaminated by noise. This will surely be the case when severe events are observed and more study is needed for stratiform cases.

How much these variables are affected by propagation effects, ground clutter, biological scatterers, chaff and clear air phenomena remain open questions, as well as how quantitavely they can improve our understanding of storms and precipitation. 


\section{References}

Cloude, S. R., Fortuny, J., Lopez, J. M., and Sieber, A. J.: Wide Band Polarimetric Radar Inversion Studies for Vegetation Layers', IEEE Transactions on Geoscience and Remote Sensing, Vol37/2 No 5, 2430-2442, September 1999.

Cloude, S. R. and Pottier, E.: A Review of Target Decomposition Theorems in Radar Polarimetry, IEEE Transactions on Geoscience and Remote Sensing, Vol. 34 No. 2, 498-518, March 1996.

Cloude, S. R. and Pottier, E.: An Entropy Based Classification Scheme for Land Applications of Polarimetric SAR, IEEETransactions on Geoscience and Remote Sensing, Vol. 35, No. 1, 6878, January 1997.

Bringi, V. N. and Chandrasekhar, V.: Polarmetric Doppler Weather Radar, University Press, 2001.

Pruppacher, H. R. and Klett, J. D.: Microphysics of Clouds and Precipitation, Second Revised and Enlarged Edition with an Introduction to Cloud Chemistry and Cloud Electricity, Kluwer Academic Publishers, 1997. 